\title{
The accuracy investigation of point coordinates' determination using a fixed basis for high-precision geodesy binding
}

\author{
Volodymyr Litynskyi ${ }^{1}$, Svyatoslav Litynskyi ${ }^{2}$, Anatolii Vivat ${ }^{*}$, Mykhailo \\ Fys $^{1}$ and Andrii Brydun ${ }^{1}$
}

\author{
${ }^{1}$ Institute of Geodesy, Lviv Polytechnic National University, 12 Bandera str., Lviv, Ukraine \\ ${ }^{2}$ Department of Programming of Ivan Franko National University of Lviv, 1 Universytetska str., Lviv, Ukraine \\ *anatolii.y.vivat@lpnu.ua
}

\begin{abstract}
Modern scanners can perform terrestrial topographic survey with resolution of $1 \mathrm{~cm}$ and accuracy of $2 \mathrm{~mm}$ in just a few minute's time, from the distance of up to 100 meters. However, for surface topographical surveying of large territories or complex industrial objects, it is necessary to conduct geodetic traverses and perform their binding to the points of the geodesic basis. One method of coordinate transferring during surveying is by using the method of inverse linear-angular intersection, which involves the measuring of the respective sides $S_{1}, S_{2}$ and the $\beta$ angle between them. This method is more precise than the classical one, which usually contains centring and reduction errors. The linear-angular intersection method can also be used for many applications in engineering geodesy, for laying geodetic traverses, and for binding to the wall based points of ground-surveying.
\end{abstract}

Key words: optimization of linear-angular measurements, linear-angular measurements, accuracy of coordinate's determination

\section{Introduction}

In the research of Lityns'kyy et al. (2014), the theoretical solution to the direct problem was found - calculation of optimal values of the measured distances for the accurate determination of the small segments' lengths. In the research of Lityns'kyy et al. (2015), the methodology was practically tested, with the electronic tacheometers (with characteristics $\left.m_{S}=2 \mathrm{~mm}, m_{\beta}=5^{\prime \prime}\right)$, determined the length of one-meter and ten-meter dimensional segments with an accuracy of 0.2-0.8 $\mathrm{mm}$. In this proposed research, the inverse problem is solved: 'Optimization of determining the coordinates of an unknown point for a fixed basis in order to provide a high-precision geodetic binding'. This allows us to design a network of reference points to obtain the highest accuracy of the position of the desired point. Depending on the distance selected between the reference points, the accuracy of determining of the position of the desired point can vary considerably for the basic indicators of accuracy of measuring lines and angles. Classically in geodesy, the method of determining the coordinates of an unknown point at measured angles and distances to known points is called reverse linear angular intersection. When measuring angles, distances and their combinations, the reverse intersections are divided into: angular, linear and angular-linear. Mathematically, the problem is unambiguously solved if two angles are measured for angular intersection, one angle and one distance for linear-angular intersection and two distances for linear intersection. Geodesically, additional (redundant) measurements are performed to assess accuracy. The form of a triangle formed during the measurement of angles, lines, angles and lines affects the accuracy of the determination of the desired point. Of particular interest is the linear-angular inter- 


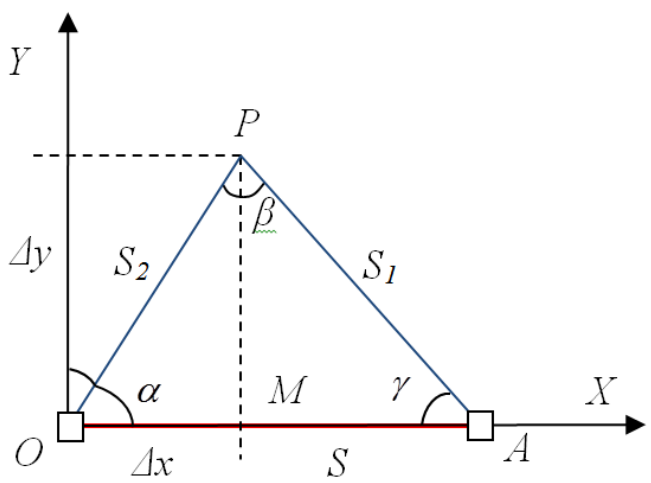

Figure 1. Regarding the theory of coordinate determination using the planar linear-angular intersection method

section, since it can use combinations of measurements.

The research of Gargula (2009) presents the theoretical and empirical studies of the accuracy of the angle determination in a triangle using the law of sines, if the angle is close to the direct one. The research is done for use in geodesy (for example, to determine the coordinates), which requires a certain level of accuracy. Several models have been researched for various triangular variants, on the basis of which a 'safe' angle was determined.

Nowadays, there are several methods for evaluating the accuracy of the inverse linear-angular intersection. We have proposed a method for determining the position of an unknown point $\mathrm{P}$, which provides an opportunity to predict the accuracy of determining its coordinates, depending on the distance between the reference points, the distance to the desired point and the accuracy of the device used.

Such studies are of interest to high-precision engineering and geodetic works, where apriori it is necessary to know the possible accuracy of the instrument's coordinates (Vivat et al., 2018) for planning the locations of the installation of an electronic tacheometer or a laser scanner.

\section{Mathematical research}

The coordinates of an unknown point $P$ are calculated as:

$$
\begin{aligned}
& x_{P}=x_{0}+\Delta x \\
& y_{P}=y_{0}+\Delta y
\end{aligned}
$$

where:

$x_{P}, y_{P}$ - coordinates of an unknown point,

$x_{0}, y_{0}$ - coordinates of a known point,

$\Delta x, \Delta y$ - increase of coordinates.

Let's find out the values of the measured parameters $S_{1}, S_{2}$ and $\beta$ when the error of the calculation of coordinates will be minimal. In this case, one side of the formed triangle $S$ is fixed (Fig. 1).

For the measured two sides of the triangle and the angle between them, the increase of coordinates $\Delta x$ and $\Delta y$ can be calculated by various methods, for example, by the law of sines and cosines, and also the Pythagorean theorem (Shkil, 2005). Let's write down these formulas in a sequential manner.

Law of sines:

$$
\begin{aligned}
& \alpha=\arcsin \left(\frac{S_{1}}{S} \sin \beta\right), \\
& \gamma=\arcsin \left(\frac{S_{2}}{S} \sin \beta\right) .
\end{aligned}
$$

Let's determine the increase of the coordinates $\Delta_{X}$ by using the value of the angles from the formulas (2) and (3):

$$
\begin{aligned}
& \Delta x=S_{2} \cos \alpha=S_{2} \sqrt{1-\frac{S_{1}^{2} \sin ^{2} \beta}{S^{2}}}=\frac{S_{2}}{S} \sqrt{S^{2}-S_{1}^{2} \sin ^{2} \beta}, \\
& \Delta x=S-S_{1} \cos \gamma=S-\frac{S_{1}}{S} \sqrt{S^{2}-S_{2}^{2} \sin ^{2} \beta} .
\end{aligned}
$$

By the Pythagorean theorem, we define the increase $\Delta x$ for the acute and obtuse angle:

$$
\Delta x=\frac{-S_{1}^{2}+S_{2}^{2}+S^{2}}{2 S} .
$$

The increase in $\Delta y$ for all cases can be determined by one formula:

$$
\Delta y=S_{2} \sin \alpha=\frac{S_{1} S_{2} \sin \beta}{S},
$$

With corresponding mean square error of:

$$
m_{y}^{2}=\frac{1}{S^{2}}\left(S_{2}^{2} \sin ^{2} \beta \cdot m_{S_{1}}^{2}+S_{1}^{2} \sin ^{2} \beta \cdot m_{S_{2}}^{2}+S_{1}^{2} S_{2}^{2} \cos ^{2} \beta \cdot m_{\beta}^{2}\right) .
$$

Additionally, from the triangle OPM we also calculate:

$$
\Delta_{y}=\sqrt{S_{2}^{2}-\left(\Delta_{x}\right)^{2}} .
$$

Therefore, it is important to choose such formulas for which there are no peculiarities and which have the slightest error in finding the coordinates of point $P$. For this, we will write down formulas of mean square errors for different cases.

Using formulas (4)-(6) gives three cases of calculation of the value $m_{x}^{2}$ :

$$
\begin{aligned}
m_{x}^{2}= & \frac{1}{S^{2}}\left(\left(S^{2}-S_{1}^{2} \sin ^{2} \beta\right) m_{S_{2}}^{2}\right. \\
& \left.+S_{2}^{2} \frac{S_{1}^{2} \sin ^{4} \beta \cdot m_{S_{1}}^{2}+S_{1}^{4} \sin ^{2} \beta \cos ^{2} \beta \cdot m_{\beta}^{2}}{S^{2}-S_{1}^{2} \sin ^{2} \beta}\right), \\
m_{x}^{2}= & \frac{1}{S^{2}}\left(\left(S^{2}-S_{2}^{2} \sin ^{2} \beta\right) m_{S_{1}}^{2}\right. \\
& \left.+S_{1}^{2} \frac{S_{2}^{2} \sin ^{4} \beta \cdot m_{S_{2}}^{2}+S_{2}^{4} \sin ^{2} \beta \cos ^{2} \beta \cdot m_{\beta}^{2}}{S^{2}-S_{2}^{2} \sin ^{2} \beta}\right), \\
m_{x}^{2}= & \frac{S_{1}^{2} m_{S_{1}}^{2}+S_{2}^{2} m_{S_{2}}^{2}}{S^{2}} .
\end{aligned}
$$
of:

Mathematically, the problem is to find the minimum error

$$
m_{P}^{2}=m_{x}^{2}+m_{y}^{2}
$$

providing

$$
S^{2}=S_{1}^{2}+S_{2}^{2}-2 S_{1} S_{2} \cos \beta,
$$

is formulated as finding the minimum of the Lagrange function:

$$
F\left(S_{1}, S_{2}, \beta\right)=m_{x}^{2}+m_{y}^{2}+\lambda\left(S_{1}^{2}+S_{2}^{2}-2 S_{1} S_{2} \cos \beta-S^{2}\right) .
$$

Analysis of the two formulas for the calculation of errors (10), (11) reveals a feature (Fig. 2), namely:

$$
\lim m_{x}^{2} \rightarrow \infty,
$$




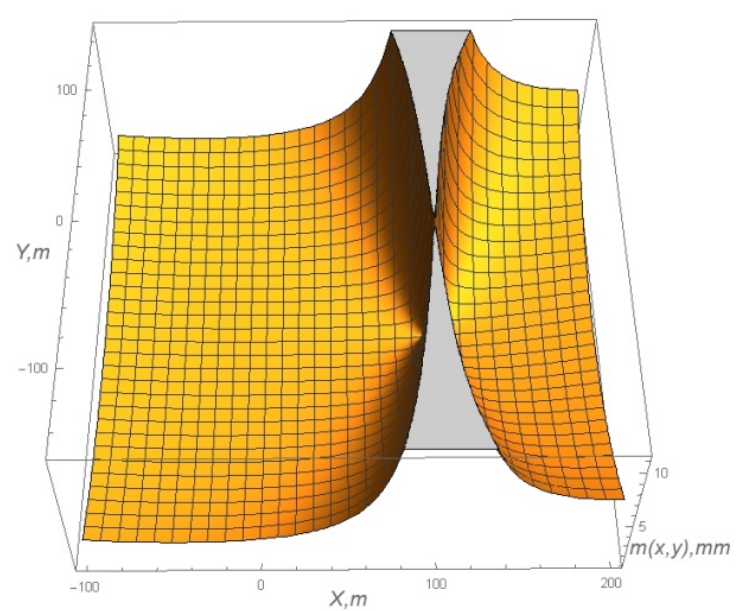

Figure 2. Peculiarity of determination of coordinates by law of sines

Table 1. The value of $m_{P}$ of nine characteristic points for Fig. 3

\begin{tabular}{crrrcr}
\hline Point & $\mathrm{x}[\mathrm{m}]$ & $\mathrm{y}[\mathrm{m}]$ & $S_{1}[\mathrm{~m}]$ & $S_{2}[\mathrm{~m}]$ & $m_{P}[\mathrm{~mm}]$ \\
\hline b1 & 0.0 & 0.0 & \multicolumn{3}{c}{$S=100 \mathrm{~m}$} \\
b2 & 0.0 & 100.0 & $m_{S}=1 \mathrm{~mm}, \mathrm{~m}_{\beta}=1^{1 "}$ \\
p1 & 0.0 & 50.0 & 50.0 & 50.0 & 1.0 \\
p2 & 50.0 & 50.0 & 70.7 & 70.7 & 1.5 \\
p3 & 100.0 & 50.0 & 111.8 & 111.8 & 2.2 \\
p4 & 100.0 & 0.0 & 141.4 & 100.0 & 2.3 \\
p5 & 0.0 & -50.0 & 150.0 & 50.0 & 1.5 \\
p6 & 0.0 & -100.0 & 200.0 & 100.0 & 2.5 \\
p7 & -100.0 & 0.0 & 141.4 & 100.0 & 2.2 \\
p8 & -100.0 & 50.0 & 111.8 & 111.8 & 2.0 \\
p9 & -50.0 & 50.0 & 70.7 & 70.7 & 1.5 \\
\hline & & & & &
\end{tabular}

for

$\sin \beta \rightarrow \frac{S}{S_{1}}\left(S_{1} \geq S, \sin \alpha \rightarrow 1, \alpha \rightarrow \frac{\pi}{2}, \Delta x \rightarrow 0, S_{1} \rightarrow S, m_{x} \rightarrow \infty\right)$

in formula (10), and

$\sin \beta \rightarrow \frac{S}{S_{2}}\left(S_{2} \geq S, \sin \gamma \rightarrow 1, \gamma \rightarrow \frac{\pi}{2}, \Delta x \rightarrow 0, S_{2} \rightarrow S, m_{x} \rightarrow \infty\right)$

in formula (11).

Also, by tabulating functions (10) and (11) it is established that for the given $S_{1}, S_{2}$, fixed basis $S$ and a definite angle $\beta$, there is no extremum by the law of cosines.

We perform the analysis of formulas $(8,12,13)$. For the graphical and tabular representation of the values of formula (13), we use the software (Wolfram Mathematics). Figures 3-5 presents the accuracy of determining the position of the point $P$, depending on the length of the base segment and the accuracy of the measurement of angles and lines. The values of the accuracy of the determination of coordinates in the characteristic points of $P_{i}$ are also given in Tables 1-3.

Table 1 gives the accuracy of determining the coordinates of the point $P$ for the characteristic discretely selected nine points for $S=100 \mathrm{~m}, m_{S}=1 \mathrm{~mm}, m_{\beta}=1$ " from Fig. 3a. Columns 4 and 5 also show the values of the formed sides $S_{1}$ and $S_{2}$.

Table2 gives the accuracy of determining the coordinates of the point $P$ for the characteristic discretely selected nine points for $S=3000 \mathrm{~m}, m_{S}=1 \mathrm{~mm}, m_{\beta}=1$ " from Fig. $4 \mathrm{a}$.

Table 3 gives the accuracy of determining the coordinates of the point $P$ for the characteristic discretely selected nine points for $S=300 \mathrm{~m}, m_{S}=0.1 \mathrm{~mm}, m_{\beta}=1$ " from Fig. $5 \mathrm{a}$.

For Figs 4 and 5 , an interesting dependence of the accuracy

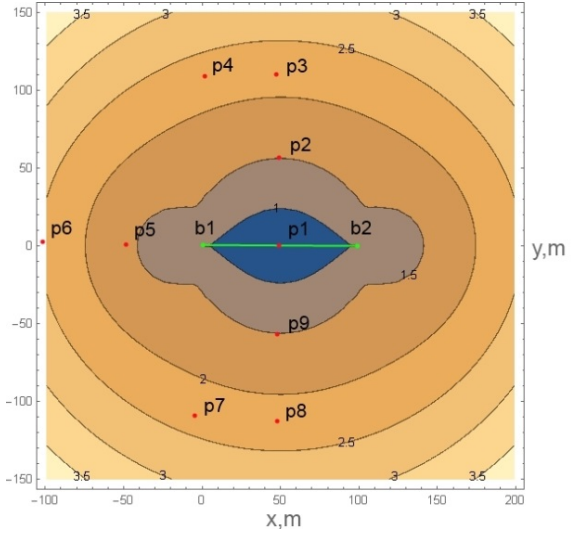

(a) $2 \mathrm{D}$ view

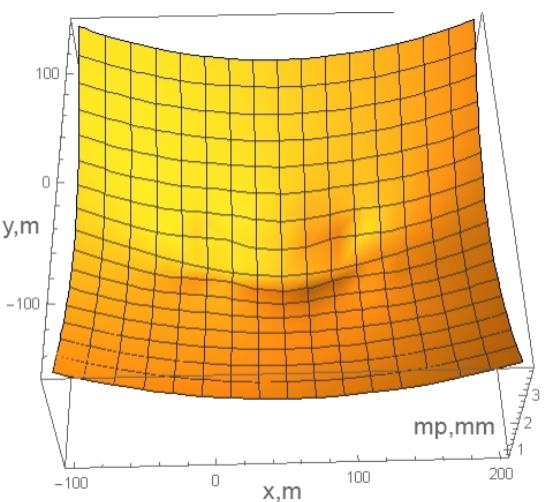

(b) $3 \mathrm{D}$ view

Figure 3. The accuracy of determining the point $P$ for $S=100 \mathrm{~m}$, $m_{S}=1 \mathrm{~mm}, m_{\beta}=1^{\prime \prime}$

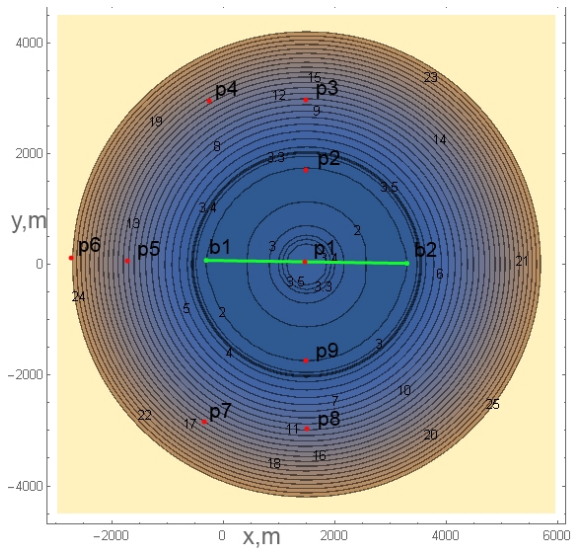

(a) $2 \mathrm{D}$ view

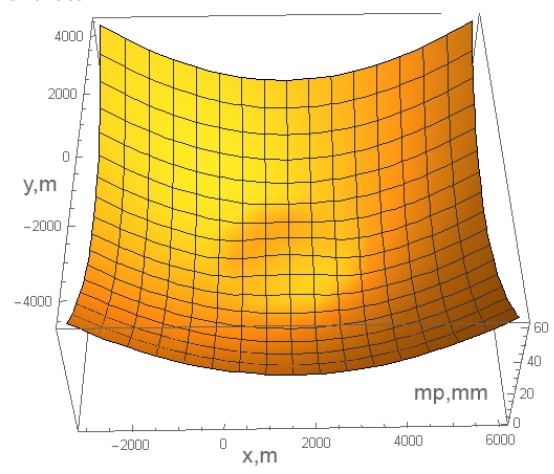

(b) $3 \mathrm{D}$ view

Figure 4. The accuracy of determining the point $P$ for $S=3000 \mathrm{~m}$, $m_{S}=1 \mathrm{~mm}, m_{\beta}=1$ 
Table 2. The value of $m_{P}$ of nine characteristic points for Fig. 4

\begin{tabular}{rrrccc}
\hline Point & $\mathrm{x}[\mathrm{m}]$ & $\mathrm{y}[\mathrm{m}]$ & $S_{1}[\mathrm{~m}]$ & $S_{2}[\mathrm{~m}]$ & $m_{P}[\mathrm{~mm}]$ \\
\hline b1 & 0.0 & 0.0 & \multicolumn{3}{c}{$S=3000 \mathrm{~m}$} \\
b2 & 0.0 & 3000.0 & $m_{S}=1 \mathrm{~mm}, m_{\beta}=1 "$ \\
p1 & 0.0 & 1500.0 & 1500.0 & 1500.0 & 3.4 \\
p2 & 1500.0 & 1500.0 & 2120.0 & 2120.0 & 2.0 \\
p3 & 3000.0 & 1500.0 & 3354.0 & 3354.0 & 13.0 \\
p4 & 3000.0 & 0.0 & 4242.0 & 3000.0 & 14.0 \\
p5 & 0.0 & -1500.0 & 4500.0 & 1500.0 & 11.0 \\
p6 & 0.0 & -3000.0 & 6000.0 & 3000.0 & 25.0 \\
p7 & -3000.0 & 0.0 & 4242.0 & 3000.0 & 14.0 \\
p8 & -3000.0 & 1500.0 & 3354.0 & 3354.0 & 12.0 \\
p9 & -1500.0 & 1500.0 & 2120.0 & 2120.0 & 2.0 \\
\hline
\end{tabular}

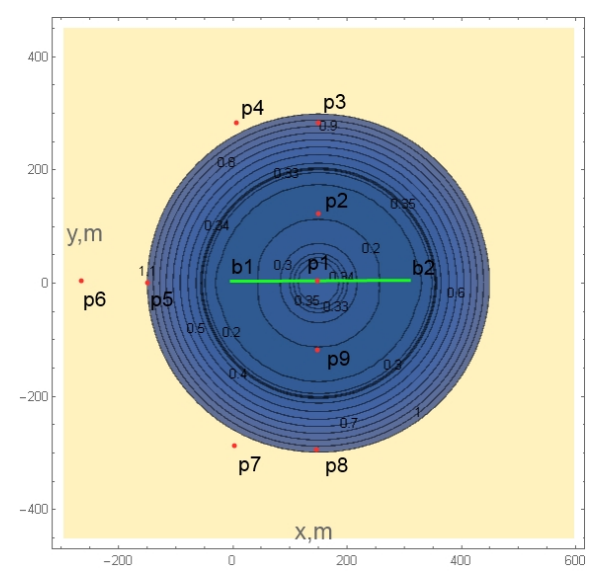

(a) $2 \mathrm{D}$ view

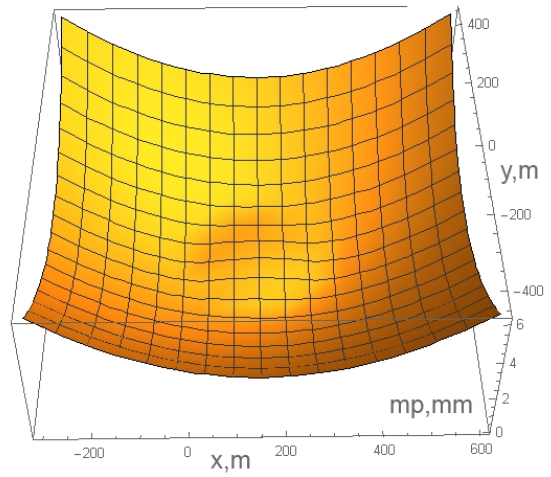

(b) $3 \mathrm{D}$ view

Figure 5. Accuracy of determining the point $P$ for $S=300 \mathrm{~m}, m_{S}=$ $0.1 \mathrm{~mm}, m_{\beta}=1$

Table 3. The value of $m_{P}$ of nine characteristic points for Fig. 5

\begin{tabular}{|c|c|c|c|c|c|}
\hline Point & $\mathrm{x}[\mathrm{m}]$ & $\mathrm{y}[\mathrm{m}]$ & $S_{1}[\mathrm{~m}]$ & $S_{2}[\mathrm{~m}]$ & $m_{P}[\mathrm{~mm}]$ \\
\hline b1 & 0.0 & 0.0 & \multicolumn{3}{|c|}{$S=300 \mathrm{~m}$} \\
\hline b2 & 0.0 & 300.0 & \multicolumn{3}{|c|}{$m_{S}=0.1 \mathrm{~mm}, m_{\beta}=1^{\prime \prime}$} \\
\hline $\mathrm{p} 1$ & 0.0 & 150.0 & 150.0 & 150.0 & 0.4 \\
\hline $\mathrm{p} 2$ & 150.0 & 150.0 & 212.0 & 212.0 & 0.2 \\
\hline p3 & 300.0 & 150.0 & 354.0 & 354.0 & 2.2 \\
\hline $\mathrm{p} 4$ & 300.0 & 0.0 & 424.0 & 300.0 & 1.3 \\
\hline p5 & 0.0 & -150.0 & 450.0 & 150.0 & 1.1 \\
\hline p6 & 0.0 & -300.0 & 600.0 & 300.0 & 2.1 \\
\hline p7 & -300.0 & 0.0 & 424.0 & 300.0 & 1.4 \\
\hline p8 & -300.0 & 150.0 & 353.0 & 353.0 & 1.1 \\
\hline p9 & -150.0 & 150.0 & 212.0 & 212.0 & 0.2 \\
\hline
\end{tabular}

of the determination of the coordinates of a point $P$ is observed. If the point $P$ is placed on a circle with a diameter equal to the base $S$, then the accuracy of the $m_{P}$ is the same for all the points that are on the diameter.

\section{The formula derive for determining the op- timal position}

Let's try to analytically derive the formula for determining the optimal position of point $P$. We will write a function for which it is necessary to determine the minimum:

$$
\begin{gathered}
F\left(S_{1}, S_{2}, \beta\right)=\frac{1}{S^{2}}\left(\left(S_{1}^{2}+S_{2}^{2} \sin ^{2} \beta\right) m_{S_{1}}^{2}+\left(S_{2}^{2}+S_{1}^{2} \sin ^{2} \beta\right) m_{S_{2}}^{2}\right. \\
\left.+\left(S_{1}^{2} S_{2}^{2} \cos ^{2} \beta\right) m_{\beta}^{2}\right)+\lambda\left(S_{1}^{2}+S_{2}^{2}-2 S_{1} S_{2} \cos \beta-S^{2}\right) .
\end{gathered}
$$

Assume that $m_{S_{1}}^{2}=m_{S_{2}}^{2}$. Accordingly, the function:

$$
\begin{aligned}
& F\left(S_{1}, S_{2}, \beta\right)=\frac{1}{S^{2}}\left(\left(S_{1}^{2}+S_{2}^{2}\right)(1\right.\left.\left.+\sin ^{2} \beta\right) m_{S_{1}}^{2}+\left(S_{1}^{2} S_{2}^{2} \cos ^{2} \beta\right) m_{\beta}^{2}\right) \\
&+\lambda\left(S_{1}^{2}+S_{2}^{2}-2 S_{1} S_{2} \cos \beta-S^{2}\right) .
\end{aligned}
$$

Let's write the conditions for the existence of an extremum:

$$
\left\{\begin{aligned}
\frac{\partial F}{\partial S_{1}}= & 2 \frac{S_{1}}{S^{2}}\left(\left(1+\sin ^{2} \beta\right) m_{S_{1}}^{2}+S_{2}^{2} \cos ^{2} \beta m_{\beta}^{2}\right) \\
& +2 \lambda\left(S_{1}-S_{2} \cos \beta\right)=0 \\
\frac{\partial F}{\partial S_{2}}= & 2 \frac{S_{2}}{S^{2}}\left(\left(1+\sin ^{2} \beta\right) m_{S_{1}}^{2}+S_{1}^{2} \cos ^{2} \beta m_{\beta}^{2}\right) \\
& +2 \lambda\left(S_{2}-S_{1} \cos \beta\right)=0 . \\
\frac{\partial F}{\partial \beta}= & \frac{\sin 2 \beta}{S^{2}}\left(\left(S_{1}^{2}+S_{2}^{2}\right) m_{S_{1}}^{2}-S_{1}^{2} S_{2}^{2} m_{\beta}^{2}\right)+2 \lambda S_{1} S_{2} \sin \beta=0 \\
S^{2}= & S_{1}^{2}+S_{2}^{2}-2 S_{1} S_{2} \cos \beta
\end{aligned}\right.
$$

Let's note $k=\frac{m_{S_{1}}^{2}}{m_{\beta}^{2}}$. Accordingly, the system of equations (18) could be rewritten as:

$$
\left\{\begin{array}{c}
\frac{S_{1} m_{\beta}^{2}}{S^{2}}\left(\left(1+\sin ^{2} \beta\right) k+S_{2}^{2} \cos ^{2} \beta\right)+\lambda\left(S_{1}-S_{2} \cos \beta\right)=0 \\
\frac{m_{\beta}^{2}}{S^{2}}\left(\left(1+\sin ^{2} \beta\right) k\left(S_{1}-S_{2}\right)-S_{1} S_{2} \cos ^{2} \beta\left(S_{1}-S_{2}\right)\right) \\
\quad+\lambda\left(S_{1}-S_{2}\right)(1+\cos \beta)=0 \\
\sin \beta\left(\frac{\cos \beta m_{\beta}^{2}}{S^{2}}\left(\left(S_{1}^{2}+S_{2}^{2}\right) k-S_{1}^{2} S_{2}^{2}\right)+\lambda S_{1} S_{2}\right)=0 \\
S^{2}=S_{1}^{2}+S_{2}^{2}-2 S_{1} S_{2} \cos \beta
\end{array}\right.
$$

or

$$
\left\{\begin{array}{l}
\frac{S_{1} m_{\beta}^{2}}{S^{2}}\left(\left(1+\sin ^{2} \beta\right) k+S_{2}^{2} \cos ^{2} \beta\right)+\lambda\left(S_{1}-S_{2} \cos \beta\right)=0 \\
\left(S_{1}-S_{2}\right)\left(m_{\beta}^{2} \frac{\left(1+\sin ^{2} \beta\right) k-S_{1} S_{2} \cos ^{2} \beta}{S^{2}}+\lambda(1+\cos \beta)\right)=0 \\
\sin \beta\left(\frac{\cos \beta m_{\beta}^{2}}{S^{2}}\left(\left(S_{1}^{2}+S_{2}^{2}\right) k-S_{1}^{2} S_{2}^{2}\right)+\lambda S_{1} S_{2}\right)=0 \\
S^{2}=S_{1}^{2}+S_{2}^{2}-2 S_{1} S_{2} \cos \beta
\end{array}\right.
$$

The second equation of the system (20) splits into two. For 
$S_{1}=S_{2}$ we have:

$$
\left\{\begin{array}{l}
S_{1}\left(\frac{m_{\beta}^{2}}{S^{2}}\left(\left(1+\sin ^{2} \beta\right) k+S_{1}^{2} \cos ^{2} \beta\right)\right)=-\lambda S_{1}(1-\cos \beta) \\
\sin \beta\left(\frac{\cos \beta m_{\beta}^{2}}{S^{2}}\left(\left(2 S_{1}^{2} k\right)-S_{1}^{4}\right)\right)=-\lambda S_{1}^{2} \sin \beta \\
S^{2}=2 S_{1}^{2}-2 S_{1}^{2} \cos \beta=0
\end{array}\right.
$$

Assuming that $\sin \beta \neq 0$ (not a degenerative case), we obtain from the first two equations of the system (21a):

$$
\frac{\left(1+\sin ^{2} \beta\right) k+S_{1}^{2} \cos ^{2} \beta}{\cos \beta\left(2 k-S_{1}^{2}\right)}=1-\cos \beta
$$

Therefore, taking into account (21c), equation (22) will have the following form:

$$
\frac{2(1-\cos \beta)\left(1+\sin ^{2} \beta\right) k+S^{2} \cos ^{2} \beta}{\cos \beta\left(2 k-\frac{S^{2}}{2(1-\cos \beta)}\right)}=2(1-\cos \beta)^{2}
$$

The solution (23) is determined by one of the approximate methods, for example, by the bisection method. The results of calculations show the existence of a minimum error value only for certain bases shown in Figures 3-5.

\section{Closing remarks}

The determination of the point coordinates for a fixed basis is possible with different formulas.

The measurement error of the calculations for some methods does not accept optimal values, but varies in the range from infinity to a fixed value.
Only the combined method of determining the coordinates can optimize the measurement error of the calculations.

It is established that the constant error of calculations is achieved when placing a device in a circle whose diameter is equal to the basis $S$.

The value of this error is determined by the formula:

$$
m^{2}\left(\frac{\pi}{2}\right)=2 m_{s}^{2} .
$$

There is a dependence between the basis and the accuracy of measurements, which is established on the basis of an approximate solution of the equation.

\section{References}

Gargula, T. (2009). A special case of the triangle solution with the law of sines in geodetic application. Modern achievements of geodesic science and production, 1(17):85-91.

Lityns'kyy, V., Fys, M., Pokotylo, I., and Lityns'kyy, S. (2014). Calculation of optimal values of measured lenghts for accurate determination of small segments. Geodesy, Cartography and Aerial Photography, 79:42-47.

Lityns'kyy, V., Vivat, A., Perii, S., and Lityns'kyy, S. (2015). Method of measuring of exemplary basis of second category for verification of electronic total stations. Geodesy, Cartography and Aerial Photography, 81:59-65.

Shkil, M. I. (2005). Matematychnyi analiz. Ch. 1. (Calculus. Part 1). Kyiv.

Vivat, A. I., Tserklevych, A. L., and Smirnova, O. M. (2018). A study of devices used for geometric parameter measurement of engineering building construction. Geodesy, Cartography, and Aerial Photography, 87:21-29, doi:10.23939/istcgcap2018.01.021. 